DEMONSTRATIO MATHEMATICA

Vol. XLII No 2009

Wen-rong Li, Sui Sun Cheng

\title{
A PICARD THEOREM FOR ITERATIVE DIFFERENTIAL EQUATIONS
}

\begin{abstract}
A Picard type existence and uniqueness theorem is established for iterative differential equations of the form $y^{\prime}(x)=f(x, y(h(x)+g(y(x))))$, a special case of which is $y^{\prime}(x)=f(x, y(y(x)))$. Such iterative differential equations can be used to model infective disease processes, pattern formation in the plane, and in investigations of dynamical systems.
\end{abstract}

\section{Introduction}

The existence and uniqueness of solutions of differential equations of the form

$$
y^{\prime}(x)=f(x, y(x))
$$

are of fundamental importance in the theory of ordinary differential equations. Therefore, there are now many existence and uniqueness theorems. Among these is the well known one that was proved by Picard's iteration method.

There are, however, plenty of differential equations that are useful in modeling natural processes, but cannot be written in the above form. One such equation

$$
y^{\prime}(x)=y(y(x))
$$

has appeared in the study of electrodynamics [1]. In [4], a similar equation

$$
y^{\prime}(x)=f(x, y(x), y(y(x)))
$$

is discussed and an existence theorem has been established under the standard initial condition

$$
y\left(x_{0}\right)=c
$$

1991 Mathematics Subject Classification: 34A12, 39B12.

Key words and phrases: iterative differential equation, existence and uniqueness theorem, Picard approximation. 
In this paper, we consider an equation

$$
y^{\prime}(x)=f(x, y(h(x)+g(y(x))))
$$

under a 'fixed point type' initial condition

$$
y\left(x_{0}\right)=x_{0}
$$

Note that a special case of (2) is

$$
y^{\prime}(x)=f(x, y(y(x)))
$$

if we take $h(x)=0$ and $g(u)=u$. Equation (2) may be called an iterative differential equation since iterates of the unknown function are involved. Such equations are important in the investigation of dynamical systems, infectious disease models, etc. Specific forms of these equations can, for example, be found in the review paper of Cheng [2].

Besides further examples of iterative equations in [1-8], here we provide an additional model that prompts us to study equations of the form (3). In pattern formation theory (such as fractal theory), a sequence of forms $\left\{F_{0}, F_{1}, F_{2}, \ldots\right\}$ may satisfy the iteration rule $F_{n+1}=H\left(F_{0}, F_{1}, \ldots, F_{n-1}\right)$. Here we consider a sequence of curves in the plane that can be described by a sequence of functions $y_{0}, y_{0}, y_{2}, \ldots$ Suppose the slope of the function $y_{k+1}$ is related to the value of $y_{k}$ at some $u$, that is,

$$
y_{k+1}^{\prime}(x)=L y_{k}(u)
$$

where $L$ is a constant, and $u$ is calculated at $y_{k-1}(x)$, that is,

$$
u=y_{k-1}(x) \text {. }
$$

The question then arises as what may the sequence $\left\{y_{0}, y_{1}, \ldots\right\}$ be. Such a family of functions is naturally called a solution of the above relations and its existence can be quite difficult. But one approach is to find a family of functions such that $y_{k}=y_{k+1}$ for all large $k$ (usually called a stationary solution). Then we are led to

$$
y^{\prime}(x)=L y(y(x)) .
$$

We will see in the last section that such a stationary family exists under the condition $y\left(x_{0}\right)=x_{0}$ and additional appropriate conditions.

Note that the condition $y\left(x_{0}\right)=x_{0}$ implies, in view of $(6)$, that

$$
y^{\prime}\left(x_{0}\right)=L x_{0},
$$

while the condition $y\left(x_{0}\right)=c$ leads us to

$$
y^{\prime}\left(x_{0}\right)=L y(c)
$$

which contains the unknown $y(c)$ ! In other words, the seemingly unpleasant condition $y\left(x_{0}\right)=x_{0}$ may in some cases match a required observation such as the slope of our curve at $x_{0}$. 


\section{Main results}

We will prove the following basic existence and uniqueness theorem for iterative functional differential equation of the form (2) based on the well known idea of Picard which was applied to (1).

TheOREM 1. Let $\Omega=\left\{(x, y)|| x-x_{0}|\leq a| y-,x_{0} \mid \leq b\right\}$. Suppose

(i) $f=f(x, y)$ is continuous on $\Omega$ with $M=\max _{(x, y) \in \Omega}|f(x, y)|$ such that

$$
|f(x, u)-f(x, v)| \leq L|u-v|,(x, u),(x, v) \in \Omega,
$$

for some $L>0$.

(ii) $h=h(x)$ is continuous on $\left[x_{0}-a, x_{0}+a\right]$ and $g=g(x)$ is continuous on $\left[x_{0}-M \delta, x_{0}+M \delta\right]$ where $\delta=\min \{a, b / M\}$ such that $(a) h\left(x_{0}\right)=\alpha x_{0}$ and $g\left(x_{0}\right)=\beta x_{0}$ where $\alpha, \beta \geq 0$ and $\alpha+\beta=1$, and (b) the functions $h(x)$ and $g(x)$ satisfy

$$
|h(u)-h(v)| \leq \alpha \lambda|u-v|, u, v \in\left[x_{0}-a, x_{0}+a\right],
$$

and

$$
|g(u)-g(v)| \leq \beta \mu|u-v|, u, v \in\left[x_{0}-M \delta, x_{0}+M \delta\right]
$$

for some $\lambda, \mu>0$.

If $\alpha \lambda+\beta \mu M \leq 1$, then equation (2) has a unique continuously differentiable solution $y=y(x)$ defined on $\left[x_{0}-\delta, x_{0}+\delta\right]$ such that $\left|y(x)-x_{0}\right| \leq M \delta$ and $y\left(x_{0}\right)=x_{0}$.

Before giving the formal proof, let us note that Theorem 1 reduces to the following special case when (3) is considered.

Corollary 1. Let $\Omega=\left\{(x, y)|| x-x_{0}|\leq a| y-,x_{0} \mid \leq b\right\}$. Suppose $f=$ $f(x, y)$ is continuous on $\Omega$ such that $M=\max _{(x, y) \in \Omega}|f(x, y)| \leq 1$ and

$$
|f(x, u)-f(x, v)| \leq L|u-v|,(x, u),(x, v) \in \Omega
$$

for some $L>0$. Then equation (3) has a unique continuously differentiable solution $y=\varphi(x)$ defined for $\left|x-x_{0}\right| \leq \delta$, where $\delta=\min \{a, b / M\}$, and satisfies $\varphi\left(x_{0}\right)=x_{0}$.

We also remark that the conditions (ii)(a) is imposed because when $h(x) \equiv 0$ and $g(x) \equiv x$, the corresponding $\alpha$ and $\beta$ are equal to 0 and 1 respectively. In other words, this condition is imposed for compatibility reasons.

Proof of Theorem 1. First note that if $y=y(x)$ is a $C^{(1)}\left[x_{0}-\delta, x_{0}+\delta\right]$ solution such that $\left|y(x)-x_{0}\right| \leq M \delta$ and $y\left(x_{0}\right)=x_{0}$, then $g(y(t))$ is well 
defined for $t \in\left[x_{0}-\delta, x_{0}+\delta\right]$. Furthermore,

$$
\begin{aligned}
\left|h(t)+g(y(t))-x_{0}\right| & \leq\left|h(t)-\alpha x_{0}+g(y(t))-\beta x_{0}\right| \\
& \leq\left|h(t)-h\left(x_{0}\right)\right|+\left|g(y(t))-g\left(x_{0}\right)\right| \\
& \leq \alpha \lambda\left|t-x_{0}\right|+\beta \mu\left|y(t)-x_{0}\right| \\
& \leq \alpha \lambda \delta+\beta \mu M \delta \leq \delta,
\end{aligned}
$$

so that $f(t, y(h(t)+g(y(t))))$ is well defined on $\left[x_{0}-\delta, x_{0}+\delta\right]$. Hence, by integrating (2), $y$ is a continuous solution of

$$
y(x)=x_{0}+\int_{x_{0}}^{x} f(t, y(h(t)+g(y(t)))) d t, x \in\left[x_{0}-\delta, x_{0}+\delta\right] .
$$

Conversely, if $y=y(x)$ is a continuous solution of the above "integral equation", then it is also a $C^{(1)}$ solution of our differential equation.

Let us define a sequence $\left\{y_{0}, y_{1}, \ldots\right\}$ of successive approximation of the desired solution of (7) by

$$
y_{0}(x)=x_{0},\left|x-x_{0}\right| \leq \delta,
$$

and for $m=0,1,2, \ldots$,

$$
y_{m+1}(x)=x_{0}+\int_{x_{0}}^{x} f\left(t, y_{m}\left(h(t)+g\left(y_{m}(t)\right)\right)\right) d t,\left|x-x_{0}\right| \leq \delta .
$$

We need to show that each $y_{i}$ is well defined and continuously differentiable on $\left[x_{0}-\delta, x_{0}+\delta\right]$.

To this end, note that $\left(x, y_{0}(x)\right)=\left(x, x_{0}\right) \in \Omega$ for $\left|x-x_{0}\right| \leq \delta$. Next,

$$
\begin{aligned}
\left|h(x)+g\left(y_{0}(x)\right)-x_{0}\right| & =\left|h(x)+g\left(x_{0}\right)-\alpha x_{0}-\beta x_{0}\right| \\
& \leq\left|h(x)-\alpha x_{0}\right|+\left|g\left(x_{0}\right)-\beta x_{0}\right| \\
& =\left|h(x)-h\left(x_{0}\right)\right| \leq \alpha \lambda\left|x-x_{0}\right| \leq \alpha \lambda \delta \leq \delta
\end{aligned}
$$

for $\left|x-x_{0}\right| \leq \delta$, as well as

$$
\begin{aligned}
\left|y_{1}(x)-x_{0}\right| & \leq\left|\int_{x_{0}}^{x} f\left(t, y_{0}\left(h(t)+g\left(y_{0}(t)\right)\right)\right) d t\right| \\
& =\left|\int_{x_{0}}^{x} f\left(t, x_{0}\right) d t\right| \leq M\left|x-x_{0}\right|
\end{aligned}
$$

for $\left|x-x_{0}\right| \leq \delta$, so that $\left(x, y_{1}(x)\right) \in \Omega$ for $\left|x-x_{0}\right| \leq \delta$. Next,

$$
\begin{aligned}
\left|h(x)+g\left(y_{1}(x)\right)-x_{0}\right| & \leq\left|h(x)-h\left(x_{0}\right)\right|+\left|g\left(y_{1}(x)\right)-g\left(x_{0}\right)\right| \\
& \leq \alpha \lambda\left|x-x_{0}\right|+\beta \mu\left|y_{1}(x)-x_{0}\right| \\
& \leq(\alpha \lambda+\beta \mu M) \delta \leq \delta
\end{aligned}
$$


for $\left|x-x_{0}\right| \leq \delta$ and

$$
\begin{aligned}
\left|y_{1}\left(h(x)+g\left(y_{1}(x)\right)\right)-x_{0}\right| & =\left|\int_{x_{0}}^{h(x)+g\left(y_{1}(x)\right)} f\left(t, y_{0}\left(h(t)+g\left(y_{0}(t)\right)\right)\right) d t\right| \\
& \leq M\left|h(x)+g\left(y_{1}(x)\right)-x_{0}\right| \\
& \leq M \delta \leq b
\end{aligned}
$$

for $\left|x-x_{0}\right| \leq \delta$, so that

$$
\left|y_{2}(x)-x_{0}\right| \leq\left|\int_{x_{0}}^{x} f\left(t, y_{1}\left(h(t)+g\left(y_{1}(t)\right)\right)\right) d t\right| \leq M\left|x-x_{0}\right| \leq M \delta \leq b
$$

for $\left|x-x_{0}\right| \leq \delta$. By induction, it is then not difficult to see that $y_{0}(x), y_{1}(x), \ldots$ are well defined and continuously differentiable on $\left[x_{0}-\delta, x_{0}+\delta\right]$, and $\left(x, y_{m}(x)\right),\left(x, y_{m}\left(h(x)+g\left(y_{m}(x)\right)\right)\right) \in \Omega$ for $x \in\left[x_{0}-\delta, x_{0}+\delta\right]$.

Next, we need to find estimates for the approximate solutions in $\left\{y_{0}, y_{1}, \ldots\right\}$. To this end, note that for $x \in\left[x_{0}-\delta, x_{0}+\delta\right]$,

$$
\left|y_{1}(x)-y_{0}(x)\right|=\left|\int_{x_{0}}^{x} f\left(t, y_{0}\left(h(t)+g\left(y_{0}(t)\right)\right)\right) d t\right| \leq M\left|x-x_{0}\right|
$$

$$
\begin{aligned}
\left|y_{2}(x)-y_{1}(x)\right| & \leq\left|\int_{x_{0}}^{x}\right|\left(f\left(t, y_{1}\left(h(t)+g\left(y_{1}(t)\right)\right)\right)-f\left(t, y_{0}\left(h(t)+g\left(y_{0}(t)\right)\right)\right)\right)|d t| \\
& \leq L\left|\int_{x_{0}}^{x}\right| y_{1}\left(h(t)+g\left(y_{1}(t)\right)\right)-y_{0}\left(h(t)+g\left(y_{0}(t)\right)\right)|d t| \\
& =L\left|\int_{x_{0}}^{x}\right| h(t)+g\left(y_{1}(t)\right) \\
& \leq L M\left|\int_{x_{0}}^{x}\right| h(t)+g\left(y_{1}(t)\right)-x_{0}|d t| \\
& \leq L M(\alpha \lambda+\beta \mu M)\left|\int_{x_{0}}^{x}\right| t-x_{0}|d t| \\
& \leq L M \frac{1}{2 !}\left|x-x_{0}\right|^{2},
\end{aligned}
$$


and

$$
\begin{aligned}
& \left|y_{2}\left(h(t)+g\left(y_{2}(t)\right)\right)-y_{1}\left(h(t)+g\left(y_{1}(t)\right)\right)\right| \\
\leq & \left|y_{2}\left(h(t)+g\left(y_{2}(t)\right)\right)-y_{2}\left(h(t)+g\left(y_{1}(t)\right)\right)\right| \\
& +\left|y_{2}\left(h(t)+g\left(y_{1}(t)\right)\right)-y_{1}\left(h(t)+g\left(y_{1}(t)\right)\right)\right| \\
= & \mid \int_{h(t)+g\left(y_{1}(t)\right)}^{h(t)+g\left(y_{2}(t)\right)} f\left(s, y_{1}\left(h(s)+g\left(y_{1}(s)\right)\right) d s \mid\right. \\
& +\left|y_{2}\left(h(t)+g\left(y_{1}(t)\right)\right)-y_{1}\left(h(t)+g\left(y_{1}(t)\right)\right)\right| \\
\leq & M\left|g\left(y_{2}(t)\right)-g\left(y_{1}(t)\right)\right|+\frac{L M}{2 !}\left|h(t)+g\left(y_{1}(t)\right)-x_{0}\right|^{2} \\
\leq & M \beta \mu\left|y_{2}(t)-y_{1}(t)\right|+\frac{L M}{2 !}(\alpha \lambda+\beta \mu M)^{2}\left|t-x_{0}\right|^{2} \\
\leq & \frac{L M}{2 !}(1+\beta \mu M)\left|t-x_{0}\right|^{2} .
\end{aligned}
$$

Hence,

$$
\begin{aligned}
\left|y_{3}(x)-y_{2}(x)\right| & \leq L\left|\int_{x_{0}}^{x}\right| y_{2}\left(h(t)+g\left(y_{2}(t)\right)\right)-y_{1}\left(h(t)+g\left(y_{1}(t)\right)\right)|d t| \\
& \leq \frac{L^{2} M}{3 !}(1+\beta \mu M)\left|x-x_{0}\right|^{3} .
\end{aligned}
$$

The same principle can now be used to show that

$$
\left|y_{k}(x)-y_{k-1}(x)\right| \leq \frac{L^{k-1} M}{k !}(1+\beta \mu M)^{k-2}\left|x-x_{0}\right|^{k}, k=1,2, \ldots,
$$

for $x \in\left[x_{0}-\delta, x_{0}+\delta\right]$.

Next, we need to show that the sequence of approximate solutions tends to a limiting function which is a solution of (7). To this end, note that the infinite series

$$
\sum_{k=1}^{\infty} \frac{L^{k-1} M}{k !}(1+\beta \mu M)^{k-2} \delta^{k}
$$

converges by means of the ratio test, we see that $y(x)=y_{0}(x)+\left(y_{1}(x)-y_{0}(x)\right)+\left(y_{2}(x)-y_{1}(x)\right)+\cdots<\infty, x \in\left[x_{0}-\delta, x_{0}+\delta\right]$, by the Weierstrass $M$-test. Thus,

$$
y_{m}=y_{0}+\left(y_{1}-y_{0}\right)+\cdots+\left(y_{m}-y_{m-1}\right)
$$

tends to a (continuous) limit function $y$ uniformly on $\left[x_{0}-\delta, x_{0}+\delta\right]$. Note that the composition $y_{m}\left(h(x)+g\left(y_{m}(x)\right)\right)$ also tends to $y(h(x)+g(y(x)))$ 
uniformly as $m$ tends to $\infty$ since

$$
\begin{aligned}
& \left|y_{m}\left(h(x)+g\left(y_{m}(x)\right)\right)-y(h(x)+g(y(x)))\right| \\
\leq & \left|y_{m}\left(h(x)+g\left(y_{m}(x)\right)\right)-y_{m}(h(x)+g(y(x)))\right| \\
& +\left|y_{m}(h(x)+g(y(x)))-y(h(x)+g(y(x)))\right| \\
= & \left|\int_{h(x)+g\left(y_{m}(x)\right)} f\left(t, y_{m-1}\left(h(t)+g\left(y_{m-1}(t)\right)\right)\right) d t\right| \\
& +\mid y_{m}(h(x)+g(y(x)) \\
\leq & M\left|g\left(y_{m}(x)\right)-g(y(x))\right|+\left|y_{m}(h(x)+g(y(x)))-y(h(x)+g(y(x)))\right| .
\end{aligned}
$$

Note further that

$$
\begin{aligned}
& \left|f\left(x, y_{m}\left(h(x)+g\left(y_{m}(x)\right)\right)\right)-f(x, y(h(x)+g(y(x))))\right| \\
\leq & L\left|y_{m}\left(h(x)+g\left(y_{m}(x)\right)\right)-y(h(x)+g(y(x)))\right|,
\end{aligned}
$$

hence

$$
f\left(x, y_{m}\left(h(x)+g\left(y_{m}(x)\right)\right)\right) \rightarrow f(x, y(h(x)+g(y(x))))
$$

uniformly on $\left[x_{0}-\delta, x_{0}+\delta\right]$ as $m \rightarrow \infty$. We may now see that

$$
\begin{aligned}
y(x) & =\lim _{m \rightarrow \infty} y_{m+1}(x) \\
& =\lim _{m \rightarrow \infty}\left\{x_{0}+\int_{x_{0}}^{x} f\left(t, y_{m}\left(h(t)+g\left(y_{m}(t)\right)\right)\right) d t\right\} \\
& =x_{0}+\int_{x_{0}}^{x} f(t, y(h(t)+g(y(t)))) d t
\end{aligned}
$$

for $x \in\left[x_{0}-\delta, x_{0}+\delta\right]$. This concludes our proof of the existence part.

To show uniqueness, let $z:\left[x_{0}-\delta, x_{0}+\delta\right] \rightarrow\left[x_{0}-b, x_{0}+b\right]$ be another solution of $(2)$ that satisfies $z\left(x_{0}\right)=x_{0}$. Then

$$
z(x)=x_{0}+\int_{x_{0}}^{x} f(t, z(h(t)+g(z(t)))) d t, x \in\left[x_{0}-\delta, x_{0}+\delta\right] .
$$

Furthermore,

$$
\left|y_{0}(x)-z(x)\right| \leq\left|\int_{x_{0}}^{x}\right| f(t, z(h(t)+g(z(t))))|d t| \leq M\left|x-x_{0}\right|,
$$




$$
\begin{aligned}
&\left|y_{1}(x)-z(x)\right| \leq L\left|\int_{x_{0}}^{x}\right| y_{0}\left(h(t)+g\left(y_{0}(t)\right)\right)-z(h(t)+g(z(t)))|d t| \\
&=L\left|\int_{x_{0}}^{x}\right| \int_{x_{0}}^{h(t)+g(z(t))} f(\xi, z(h(\xi)+g(z(\xi)))) d \xi|d t| \\
& \leq L M\left|\int_{x_{0}}^{x}\right| h(t)+g(z(t))-x_{0}|d t| \\
& \leq L M(\alpha \lambda+\beta \mu M)\left|\int_{x_{0}}^{x}\right| t-x_{0}|d t| \\
& \leq \frac{L M}{2 !}\left|x-x_{0}\right|^{2}, \\
& \mid y_{1}\left(h(t)+g\left(y_{1}(t)\right)-z(h(t)+g(z(t)) \mid\right. \\
& \leq \mid y_{1}\left(h(t)+g\left(y_{1}(t)\right)-y_{1}(h(t)+g(z(t)) \mid\right. \\
&+\mid y_{1}(h(t)+g(z(t))-z(h(t)+g(z(t)) \mid \\
& \leq M\left|g\left(y_{1}(t)\right)-g(z(t))\right|+\frac{L M}{2 !}(\alpha \lambda+\beta \mu M)\left|h(t)+g(z(t))-x_{0}\right|^{2} \\
& \leq M \beta \mu\left|y_{1}(t)-z(t)\right|+\frac{L M}{2 !}\left|t-x_{0}\right|^{2}
\end{aligned}
$$

and

$$
\begin{aligned}
\left|y_{2}(x)-z(x)\right| & \leq L\left|\int_{x_{0}}^{x}\right| y_{1}\left(h(t)+g\left(y_{1}(t)\right)\right)-z(h(t)+g(z(t)))|d t| \\
& \leq \frac{L^{2} M}{2 !}(1+\beta \mu M)\left|x-x_{0}\right|^{3}
\end{aligned}
$$

for $x \in\left[x_{0}-\delta, x_{0}+\delta\right]$. By induction, we may then show that

$$
\left|y_{n}(x)-z(x)\right| \leq \frac{L^{n} M}{(n+1) !}(1+\beta \mu M)^{n-1}\left|x-x_{0}\right|^{n+1}, n=1,2, \ldots .
$$

Since (9) converges, we may then see that $y_{n}$ converges to $z$ uniformly on $\left[x_{0}-\delta, x_{0}+\delta\right]$ as $n \rightarrow \infty$. This implies $y(x)=z(x)$ for $x \in\left[x_{0}-\delta, x_{0}+\delta\right]$. The uniqueness part is also proved.

\section{Examples}

We now consider three examples to illustrate our results.

EXAMPLE 1. First consider the equation

$$
y^{\prime}(x)=L y(y(x))
$$


where $L>0$. Since $f(x, y)=L y$, we see that $|f(x, u)-f(x, v)|=L|u-v|$ holds for arbitrary $(x, u)$ and $(x, v)$. Next let $x_{0} \in[0,1 / L]$. Then

$$
|f(x, y)|=L|y| \leq 1
$$

for $\left|x-x_{0}\right| \leq a \leq 1 / L-x_{0}$ and $\left|y-x_{0}\right| \leq 1 / L-x_{0}$. By Corollary 1 , (10) has a unique continuously differentiable solution $y=y(x)$ defined on $\left[x_{0}-\delta, x_{0}+\delta\right]$ and satisfies $y\left(x_{0}\right)=x_{0}$, where

$$
\delta=\min \left(a, \frac{1}{L}-x_{0}\right) .
$$

EXAMPLE 2. Consider the equation

$$
y^{\prime}(x)=f(x, y(y(x)))=x^{2}+(y(y(x)))^{2},
$$

where $f(x, y)=x^{2}+y^{2}, x_{0}=0$ and $\Omega=\left\{(x, y) \in R^{2}|| x|\leq 1 / 2| y \mid, \leq 1 / 2\right\}$. Then

$$
|f(x, u)-f(x, v)|=|u+v||u-v| \leq|u-v|,(x, u),(x, v) \in \Omega,
$$

and

$$
M=\max _{(x, y) \in \Omega}|f(x, y)|=\frac{1}{2},
$$

and $\delta=1 / 2$. Thus our equation (11) has a unique continuously differentiable solution $y=y(x)$ defined on $[-1 / 2,1 / 2]$ which satisfies $y(0)=0$.

Next, we observe that in the previous development, we assume that the independent and dependent variables are in the real domain. However, the same arguments hold when the variables are complex valued as long as we change the corresponding intervals with centers into balls. For instance, Corollary 1 remains the same in form if we interpret $x, y, f, u, v$ as complex variables.

EXAMPLE 3. In the modified Corollary, the assumption $M \leq 1$ is sufficient but not necessary. As an example, consider the equation

$$
y^{\prime}(z)=y(y(z)) \text {. }
$$

Let us look for solutions of the form [3]

$$
y(z)=\beta z^{\gamma} .
$$

After substituting it into (12), we see that

$$
\begin{array}{r}
\gamma^{2}-\gamma+1=0, \\
\beta^{\gamma}=\gamma,
\end{array}
$$

which lead us to

$$
y_{+}(z)=\beta_{+} z^{\gamma_{+}} \text {and } y_{-}(z)=\beta_{-} z^{\gamma_{-}}
$$


where

$$
\gamma_{ \pm}=\frac{1 \pm \sqrt{3} i}{2}
$$

Note that $\left|\gamma_{+}\right|=1$, and $y_{+}\left(\gamma_{+}\right)=\gamma_{+}$. Hence $y_{+}(z)$ is a continuously differentiable solution of (12) for $\left|z-\gamma_{+}\right| \leq 1$ that satisfies $y_{+}\left(\gamma_{+}\right)=\gamma_{+}$. However,

$$
\max _{\left|z-\gamma_{+}\right| \leq 1,\left|w-\gamma_{+}\right| \leq 1}|f(z, w)|=\max _{\left|w-\gamma_{+}\right| \leq 1}|w|>1
$$

\title{
References
}

[1] E. Eder, The functional differential equation $x^{\prime}(t)=x(x(t))$, J. Differential Equations 54 (1984), 390-400.

[2] S. S. Cheng, Smooth Solutions of Iterative Functional Differential Equations, 2004Dynamical Systems and Application, eds. H. Akca, A. Boucherif and V. Covachev, GBS Publishers \& Distributions, 2005.

[3] W. R. Li, S. S. Cheng, T. T. Lu, Closed form solutions of iterative functional differential equations, Appl. Math. E-Notes 1 (2001), 1-4.

[4] A. Pelczar, On some iterative differential equations I, Zeszyty Naukowe Uniwersytetu Jagiellońskiego, Prace Matematyczne 12 (1968), 53-56.

[5] R. D. Driver, A two-body problem of classical electrodynamics: the one-dimensional case, Ann. Physics 21 (1963), 122-142.

[6] R. D. Driver, Can the future influence the present?, Phys. Rev. D. 19 (4) (1979), 1098-1107.

[7] K. L. Cooke, Functional differential systems: some models and perturbation problems, Inter. Symp. Diff. Eqs. Dynamical Systems, Puerto Rico, 1965.

[8] W. X. Yang, W. G. Ge, Periodic solutions for the differential-iterative equation $\dot{x}+$ $g(x(x))=p(t)$ (in Chinese), J. Beijing Inst. Technol. (Chin. Ed.) 22 (5) (2002), no. 5, $537-539$.

\author{
Wen-rong Li \\ DEPARTMENT OF MATHEMATICS \\ BINZHOU UNIVERSITY \\ BINZHOU, SHANGDONG 256600, P.R. CHINA \\ Sui Sun Cheng \\ DEPARTMENT OF MATHEMATICS \\ TSING HUA UNIVERSITY \\ HSINCHU, TAIWAN 30043, R. O. CHINA
}

Received July 8, 2008. 